\title{
ZEROS OF RANDOM POLYNOMIALS ON $\mathbb{C}^{m}$
}

\author{
Thomas Bloom and Bernard Shiffman
}

\begin{abstract}
For a regular compact set $K$ in $\mathbb{C}^{m}$ and a measure $\mu$ on $K$ satisfying the Bernstein-Markov inequality, we consider the ensemble $\mathcal{P}_{N}$ of polynomials of degree $N$, endowed with the Gaussian probability measure induced by $L^{2}(\mu)$. We show that for large $N$, the simultaneous zeros of $m$ polynomials in $\mathcal{P}_{N}$ tend to concentrate around the Silov boundary of $K$; more precisely, their expected distribution is asymptotic to $N^{m} \mu_{e q}$, where $\mu_{e q}$ is the equilibrium measure of $K$. For the case where $K$ is the unit ball, we give scaling asymptotics for the expected distribution of zeros as $N \rightarrow \infty$.
\end{abstract}

\section{Introduction}

A classical result due to Hammersley [Ha] (see also [SV]), loosely stated, is that the zeros of a random complex polynomial

$$
f(z)=\sum_{j=0}^{N} c_{j} z^{j}
$$

mostly tend towards the unit circle $|z|=1$ as the degree $N \rightarrow \infty$, when the coefficients $c_{j}$ are independent complex Gaussian random variables of mean zero and variance one. In this paper, we will prove a multivariable result (Theorem 3.1), a special case (Example 3.5) of which shows, loosely stated, that the common zeros of $m$ random complex polynomials in $\mathbb{C}^{m}$,

$$
f_{k}(z)=\sum_{|J| \leq N} c_{J}^{k} z_{1}^{j_{1}} \cdots z_{m}^{j_{m}} \quad \text { for } k=1, \ldots, m
$$

tend to concentrate on the product of the unit circles $\left|z_{j}\right|=1(j=1, \ldots, m)$ as $N \rightarrow \infty$, when the coefficients $c_{J}^{k}$ are i.i.d. complex Gaussian random variables.

The following is our basic setting: We let $K$ be a compact set in $\mathbb{C}^{m}$ and let $\mu$ be a Borel probability measure on $K$. We assume that $K$ is non-pluripolar and we let $V_{K}$ be its pluricomplex Green function. We also assume that $K$ is regular (i.e., $V_{K}=V_{K}^{*}$ ) and that $\mu$ satisfies the Bernstein-Markov inequality (see $\S 2$ ). We give the space $\mathcal{P}_{N}$ of holomorphic polynomials of degree $\leq N$ on $\mathbb{C}^{m}$ the Gaussian probability measure $\gamma_{N}$ induced by the Hermitian inner product

$$
(f, g)=\int_{K} f \bar{g} d \mu .
$$

Received by the editors May 29, 2006.

2000 Mathematics Subject Classification. 32A60, 32U35, 60D05, 60G99.

Research partially supported by NSERC (first author) and NSF grant DMS-0100474 (second author). 
The Gaussian measure $\gamma_{N}$ can be described as follows: We write $f=\sum_{j=1}^{d(N)} c_{j} p_{j}$, where $\left\{p_{j}\right\}$ is an orthonormal basis of $\mathcal{P}_{N}$ with respect to $(3)$ and $d(N)=\operatorname{dim} \mathcal{P}_{N}=$ $\left(\begin{array}{c}N+m \\ m\end{array}\right)$. Identifying $f \in \mathcal{P}_{N}$ with $c=\left(c_{1}, \ldots, c_{d(N)}\right) \in \mathbb{C}^{d(N)}$, we have

$$
d \gamma_{N}(s)=\frac{1}{\pi^{d(N)}} e^{-|c|^{2}} d c
$$

(The measure $\gamma_{N}$ is independent of the choice of orthonormal basis $\left\{p_{j}\right\}$.) In other words, a random polynomial in the ensemble $\left(\mathcal{P}_{N}, \gamma_{N}\right)$ is a polynomial $f=\sum_{j} c_{j} p_{j}$, where the $c_{j}$ are independent complex Gaussian random variables with mean 0 and variance 1 .

Our main result, Theorem 3.1, gives asymptotics for the expected zero current of $k$ i.i.d. random polynomials with respect to the Gaussian probability measure induced by (3) (where $1 \leq k \leq m$ ). In particular, the expected distribution $\mathbf{E}\left(Z_{f_{1}, \ldots, f_{m}}\right)$ of simultaneous zeros of $m$ independent random polynomials in $\left(\mathcal{P}_{N}, \gamma_{N}\right)$ has the asymptotics

$$
\frac{1}{N^{m}} \mathbf{E}\left(Z_{f_{1}, \ldots, f_{m}}\right) \rightarrow \mu_{e q} \quad \text { weak } k^{*}
$$

where $\mu_{e q}=\left(\frac{i}{\pi} \partial \bar{\partial} V_{K}\right)^{m}$ is the equilibrium measure of $K$ (see (8) below). Here, $\mathbf{E}(X)$ denotes the expected value of a random variable $X$.

We now describe some recent related results on expected distributions of zeros. The one-dimensional case of (4) was given in [Bl2], which generalized the results in [SZ2] for the case where $K$ is a real-analytic domain in $\mathbb{C}$ (or its boundary). After this paper was written, generalizations of (4) to weighted equilibrium measures were given in [Bl3], and generalizations to equilibrium measures on pseudoconcave domains in compact Kähler manifolds were given by R. Berman [Be]. It was also shown in [Bl3] that (4) holds for certain non-Gaussian random polynomials on $\mathbb{C}$. Results on the distribution of zeros of polynomials on $\mathbb{C}$ with random real coefficients were given by Shepp-Vanderbei [SV], Ibragimov-Zeitouni [IZ] and others.

The reader may notice that the distributions of zeros for the measures on $\mathcal{P}_{N}$ considered here are quite different from those of the $\mathrm{SU}(m+1)$ ensembles studied, for example, in [SZ1, SZ4, BSZ1, BSZ2, DS]. The Gaussian measure on the SU $(m+1)$ polynomials is based on the inner product

$$
\langle f, g\rangle_{N}=\int_{S^{2 m+1}} F_{N} \overline{G_{N}},
$$

where $F_{N}, G_{N} \in \mathbb{C}\left[z_{0}, z_{1}, \ldots, z_{m}\right]$ denote the degree $N$ homogenizations of $f$ and $g$ respectively. It follows easily from the $\mathrm{SU}(m+1)$-invariance of the inner product that the expected distribution of simultaneous zeros equals $\frac{N^{m}}{\pi^{m}} \omega^{m}$ (exactly), where $\omega$ is the Fubini-Study Kähler form (on $\mathbb{C}^{m} \subset \mathbb{C P}^{m}$ ). We note that, unlike (3), this inner product depends on $N$; indeed, $\left\|z^{J}\right\|_{N}^{2}=\frac{m !(N-|J|) ! j_{1} ! \cdots j_{m} !}{(N+m) !}[\mathrm{SZ} 1,(30)]$.

In this paper, we also give scaling limits for the expected zero density in the case of the unit ball in $\mathbb{C}^{m}$ (Theorem 4.1). An open problem is to find scaling limits for more general sets in $\mathbb{C}^{m}$. 


\section{Background}

We let $\mathcal{L}$ denote the Lelong class of plurisubharmonic (PSH) functions on $\mathbb{C}^{m}$ of at most logarithmic growth at $\infty$. That is

$$
\mathcal{L}:=\left\{u \in \operatorname{PSH}\left(\mathbb{C}^{m}\right) \mid u(z) \leq \log ^{+}\|z\|+O(1)\right\}
$$

For $K$ a compact subset of $\mathbb{C}^{m}$, we define its pluricomplex Green function $V_{K}(z)$ via

$$
V_{K}(z)=\sup \{u(z) \mid u \in \mathcal{L}, u \leq 0 \text { on } K\} .
$$

We will assume $K$ is regular, that is by definition, $V_{K}$ is continuous on $\mathbb{C}^{m}$ (and so $V_{K}=V_{K}^{*}$, its uppersemicontinuous regularization). The function $V_{K}$ is a locally bounded PSH function on $\mathbb{C}^{m}$ and, in fact

$$
V_{K}-\log ^{+}\|z\|=O(1) .
$$

By a basic result of Bedford and Taylor [BT1] (see [Kl]), the complex Monge-Ampère operator $\left(d d^{c}\right)^{m}=(2 i \partial \bar{\partial})^{m}$ is defined on any locally bounded PSH function $\mathbb{C}^{m}$ and in particular on $V_{K}$. The equilibrium measure of $K$ [BT1, BT2, Le, NZ, Ze] (see also $[\mathrm{Kl}$, Cor. 5.5.3]) is defined by

$$
\mu_{e q}(K):=\left(\frac{i}{\pi} \partial \bar{\partial} V_{K}\right)^{m}
$$

Since $V_{K}$ satisfies (7), it is a positive Borel measure, here normalized to have mass 1. The support of the measure $\mu_{e q}(K)$ is the Silov boundary of $K$ for the algebra of entire analytic functions [BT2]. In one variable, i.e. $K \subset \mathbb{C}, V_{K}$ is the Green function of the unbounded component of $\mathbb{C} \backslash K$ with a logarithmic pole at $\infty$, and $\mu_{e q}(K)=\frac{1}{2 \pi} \Delta V_{K}$, where $\Delta$ is the Laplacian [Ra].

Let $\mu$ be a finite positive Borel measure on $K$. The measure $\mu$ is said to satisfy a Bernstein-Markov (BM) inequality, if, for each $\varepsilon>0$ there is a constant $C=C(\varepsilon)>0$ such that

$$
\|p\|_{K} \leq C e^{\varepsilon \operatorname{deg}(p)}\|p\|_{L^{2}(\mu)}
$$

for all holomorphic polynomials $p$. Essentially, the BM inequality says that the $L^{2}$ norms and the sup norms of a sequence of holomorphic polynomials of increasing degrees are "asymptotically equivalent".

The question arises as to which measures actually satisfy the BM inequality. It is a result of Nguyen-Zeriahi [NZ] combined with [Kl, Cor. 5.6.7] that for $K$ regular, $\mu_{e q}(K)$ satisfies BM. This fact is used in Examples 3.5-3.6. In [B11, Theorem 2.2], a "mass-density" condition for a measure to satisfy BM was given. (See also [BL].)

Our proof uses the probabilistic Poincaré-Lelong formula for the zeros of random functions (Proposition 2.1 below). Considering a slightly more general situation, we let $g_{1}, \ldots, g_{d}$ be polynomials with no common zeros on a domain $U \subset \mathbb{C}^{m}$. (We are interested in the case where $U=\mathbb{C}^{m}$ and $\left\{g_{j}\right\}$ is an orthonormal basis of $\mathcal{P}_{N}$ with respect to the inner product (3), as discussed above.) We let $\mathcal{F}$ denote the ensemble of random polynomials of the form $f=\sum c_{j} g_{j}$, where the $c_{j}$ are independent complex Gaussian random variables with mean 0 and variance 1 . We consider the Szegö kernel

$$
S_{\mathcal{F}}(z, w)=\sum_{j=1}^{d} g_{j}(z) \overline{g_{j}(w)} .
$$


For the case where the $g_{j}$ are orthonormal with respect to an inner product on $\mathcal{O}(U)$, $S_{\mathcal{F}}(z, w)$ is the kernel for the orthogonal projection onto the span of the $g_{j}$.

Under the assumption that the $g_{j}$ have no common zeros, it is easily shown using Sard's theorem (or Bertini's theorem) that for almost all $f_{1}, \ldots, f_{k} \in \mathcal{F}$, the differentials $d f_{1}, \ldots, d f_{k}$ are linearly independent at all points of the zero set

$$
\operatorname{loc}\left(f_{1}, \ldots, f_{k}\right):=\left\{z \in U: f_{1}(z)=\cdots=f_{k}(z)=0\right\} .
$$

This condition implies that the complex hypersurfaces $\operatorname{loc}\left(f_{j}\right)$ are smooth and intersect transversely, and hence $\operatorname{loc}\left(f_{1}, \ldots, f_{k}\right)$ is a codimension $k$ complex submanifold of $U$. We then let $Z_{f_{1}, \ldots, f_{k}} \in \mathcal{D}^{\prime k, k}(U)$ denote the current of integration over $\operatorname{loc}\left(f_{1}, \ldots, f_{k}\right)$ :

$$
\left(Z_{f_{1}, \ldots, f_{k}}, \varphi\right)=\int_{\operatorname{loc}\left(f_{1}, \ldots, f_{k}\right)} \varphi, \quad \varphi \in \mathcal{D}^{m-k, m-k}(U) .
$$

We shall use the following Poincaré-Lelong formula:

Proposition 2.1. The expected zero current of $k$ independent random polynomials $f_{1}, \ldots, f_{k} \in \mathcal{F}$ is given by

$$
\mathbf{E}\left(Z_{f_{1}, \ldots, f_{k}}\right)=\left(\frac{i}{2 \pi} \partial \bar{\partial} \log S_{\mathcal{F}}(z, z)\right)^{k} .
$$

A proof of Proposition 2.1 (for sections of holomorphic line bundles) can be found in [SZ4]. The codimension $k=1$ case was given in [SZ1]. The general case follows from the codimension 1 case together with the fact that

$$
\mathbf{E}\left(Z_{f_{1}, \ldots, f_{k}}\right)=\mathbf{E}\left(Z_{f_{1}}\right) \wedge \cdots \wedge \mathbf{E}\left(Z_{f_{k}}\right)=\mathbf{E}\left(Z_{f}\right)^{k}
$$

which is a consequence of the independence of the $f_{j}$. The wedge product of currents is not always defined, but $Z_{f_{1}} \wedge \cdots \wedge Z_{f_{k}}$ is almost always defined (and equals $Z_{f_{1}, \ldots, f_{k}}$ whenever the hypersurfaces $\operatorname{loc}\left(f_{j}\right)$ are smooth and intersect transversely), and a short argument given in [SZ3] or [SZ4] yields (10). The point case $k=m$ of Proposition 2.1 was given by Edelman-Kostlan [EK, Th. 8.1]. We note that the expectations in (10) are smooth forms.

\section{Asymptotics of expected zero currents}

We now state our main result on the expected distribution of simultaneous zeros of random polynomials orthonormalized on a compact set:

Theorem 3.1. Let $\mu$ be a Borel probability measure on a regular compact set $K \subset \mathbb{C}^{m}$, and suppose that $(K, \mu)$ satisfies the Bernstein-Markov inequality. Let $1 \leq k \leq m$, and let $\left(\mathcal{P}_{N}^{k}, \gamma_{N}^{k}\right)$ denote the ensemble of $k$-tuples of i.i.d. Gaussian random polynomials of degree $\leq N$ with the Gaussian measure $d \gamma_{N}$ induced by $L^{2}(\mu)$. Then

$$
\frac{1}{N^{k}} \mathbf{E}_{\gamma_{N}^{k}}\left(Z_{f_{1}, \ldots, f_{k}}\right) \rightarrow\left(\frac{i}{\pi} \partial \bar{\partial} V_{K}\right)^{k} \quad \text { weak } k^{*}, \quad \text { as } N \rightarrow \infty,
$$

where $V_{K}$ is the pluricomplex Green function of $K$ with pole at infinity. 
Here we say that a sequence $T_{N}$ of currents of order 0 converges weak ${ }^{*}$ to $T$ if $\left(T_{N}, \varphi\right) \rightarrow(T, \varphi)$ for all compactly supported test forms $\varphi$ with continuous coefficients. Using general variance estimates given in [Sh] together with Theorem 3.1, one can show that with probability one, a sequence $\left\{f_{1}^{N}, \ldots, f_{k}^{N}\right\}_{N=1,2, \ldots}$ of $k$-tuples of random polynomials of increasing degree satisfies:

$$
\frac{1}{N^{k}} Z_{f_{1}^{N}, \ldots, f_{k}^{N}} \rightarrow\left(\frac{i}{\pi} \partial \bar{\partial} V_{K}\right)^{k} \quad \text { weak } k^{*}
$$

(See [Sh]; the one-dimensional case of (11) was given in [Bl2] using potential theory.)

To prove Theorem 3.1, we consider the Szegö kernels

$$
S_{N}(z, w):=S_{\left(\mathcal{P}_{N}, \gamma_{N}\right)}(z, w)=\sum_{j=1}^{d(N)} p_{j}(z) \overline{p_{j}(w)}
$$

where $\left\{p_{j}\right\}$ is an $L^{2}(\mu)$-orthonormal basis for $\mathcal{P}_{N}$. Our proof is based on approximating the extremal function $V_{K}$ by the (normalized) logarithms of the Szegö kernels $S_{N}(z, z)$ (Lemma 3.4) and then applying the Poincaré-Lelong formula of Proposition 2.1 .

We begin by considering the polynomial suprema

$$
\Phi_{N}^{K}(z)=\sup \left\{|f(z)|: f \in \mathcal{P}_{N},\|f\|_{K} \leq 1\right\} .
$$

Since $\frac{1}{N} \log f \in \mathcal{L}$, for $f \in \mathcal{P}_{N}$, it is clear that $\frac{1}{N} \log \Phi_{N}^{K} \leq V_{K}$, for all $N$. Pioneering work of Zaharjuta [Za] and Siciak [Si1, Si2] established the convergence of $\frac{1}{N} \log \Phi_{N}^{K}$ to $V_{K}$. The uniform convergence when $K$ is regular seems not to have been explicitly stated and we give the proof below.

Lemma 3.2. Let $K$ be a regular compact set in $\mathbb{C}^{m}$. Then

$$
\frac{1}{N} \log \Phi_{N}^{K}(z) \rightarrow V_{K}(z)
$$

uniformly on compact subsets of $\mathbb{C}^{m}$.

Proof. We first note that $1 \leq \Phi_{j} \leq \Phi_{j} \Phi_{k} \leq \Phi_{j+k}$, for $j, k \geq 0$. By a result of Siciak [Si1] and Zaharjuta [Za] (see [Kl, Theorem 5.1.7]),

$$
V_{K}(z)=\lim _{N \rightarrow \infty} \frac{1}{N} \log \Phi_{N}^{K}(z)=\sup _{N} \frac{1}{N} \log \Phi_{N}^{K}(z)
$$

for all $z \in \mathbb{C}^{m}$.

We use the regularity of $K$ to show that the convergence is uniform: let

$$
\psi_{N}=\frac{1}{N} \log \Phi_{N}^{K} \geq 0 .
$$

Thus for $N, k \geq 1, j \geq 0$, we have

$$
N k \psi_{N k}+j \psi_{j} \leq(N k+j) \psi_{N k+j} .
$$

Since $\psi_{N} \leq \psi_{N k}$, we then obtain the inequality

$$
\psi_{N k+j} \geq \frac{N k}{N k+j} \psi_{N}+\frac{j}{N k+j} \psi_{j} \geq \frac{N k}{N k+j} \psi_{N} .
$$


Fix $\varepsilon>0$. For each $a \in \mathbb{C}^{m}$, we choose $N_{a} \in \mathbb{Z}^{+}$such that

$$
V_{K}(a)-\psi_{N_{a}}(a)<\varepsilon \quad \text { and } \quad \frac{V_{K}(a)}{N_{a}}<\varepsilon,
$$

and then choose a neighborhood $U_{a}$ of $a$ such that

$$
\left|V_{K}(z)-V_{K}(a)\right|<\varepsilon, \quad \psi_{N_{a}}(z) \geq \psi_{N_{a}}(a)-\varepsilon, \quad \frac{V_{K}(z)}{N_{a}}<\varepsilon, \quad \text { for } z \in U_{a} .
$$

Now let $N \geq N_{a}^{2}$, and write $N=N_{a} k+j$, where $k \geq N_{a}, 0 \leq j<N_{a}$. By (13)-(14), we have

(15) $0 \leq V_{K}-\psi_{N} \leq V_{K}-\frac{N_{a} k}{N_{a} k+j} \psi_{N_{a}} \leq V_{K}-\frac{N_{a}}{N_{a}+1} \psi_{N_{a}} \leq V_{K}-\psi_{N_{a}}+\frac{1}{N_{a}+1} V_{K}$.

Hence, for all $N \geq N_{a}^{2}$ and for all $z \in U_{a}$, we have

$$
\begin{aligned}
0 & \leq V_{K}(z)-\psi_{N}(z) \\
& <V_{K}(z)-\psi_{N_{a}}(z)+\varepsilon \\
& =\left[V_{K}(a)-\psi_{N_{a}}(a)\right]+\left[V_{K}(z)-V_{K}(a)\right]+\left[\psi_{N_{a}}(a)-\psi_{N_{a}}(z)\right]+\varepsilon \\
& <4 \varepsilon .
\end{aligned}
$$

Hence for each compact $A \subset \mathbb{C}^{m}$, we can cover $A$ with finitely many $U_{a_{i}}$, so that we have by (16),

$$
\left\|V_{K}-\psi_{N}\right\|_{A} \leq 4 \varepsilon \quad \forall N \geq \max _{i} N_{a_{i}}^{2}
$$

Lemma 3.3. For all $\varepsilon>0$, there exists $C=C_{\varepsilon}>0$ such that

$$
\frac{1}{d(N)} \leq \frac{S_{N}(z, z)}{\Phi_{N}^{K}(z)^{2}} \leq C e^{\varepsilon N} d(N) .
$$

Proof. Let $f \in \mathcal{P}_{N}$ with $\|f\|_{K} \leq 1$. Then

$$
\begin{aligned}
|f(z)| & =\left|\int_{K} S_{N}(z, w) f(w) d \mu(w)\right| \leq \int_{K}\left|S_{N}(z, w)\right| d \mu(w) \\
& \leq \int_{K} S_{N}(z, z)^{\frac{1}{2}} S_{N}(w, w)^{\frac{1}{2}} d \mu(w)=S_{N}(z, z)^{\frac{1}{2}}\left\|S_{N}(w, w)^{\frac{1}{2}}\right\|_{L^{1}(\mu)} \\
& \leq S_{N}(z, z)^{\frac{1}{2}}\|1\|_{L^{2}(\mu)}\left\|S_{N}(w, w)^{\frac{1}{2}}\right\|_{L^{2}(\mu)}=S_{N}(z, z)^{\frac{1}{2}} d(N)^{\frac{1}{2}} .
\end{aligned}
$$

Taking the supremum over $f \in \mathcal{P}_{N}$ with $\|f\|_{K} \leq 1$, we obtain the left inequality of the lemma.

To verify the right inequality, we let $\left\{p_{j}\right\}$ be a sequence of $L^{2}(\mu)$-orthonormal polynomials, obtained by applying Gram-Schmid to a sequence of monomials of nondecreasing degree, so that $\left\{p_{1}, \ldots p_{d(N)}\right\}$ is an orthonormal basis of $\mathcal{P}_{N}$ (for each $N \in \mathbb{Z}^{+}$). By the Bernstein-Markov inequality (9), we have

$$
\left\|p_{j}\right\|_{K} \leq C e^{\varepsilon \operatorname{deg} p_{j}}
$$

and hence

$$
\left|p_{j}(z)\right| \leq\left\|p_{j}\right\|_{K} \Phi_{\operatorname{deg} p_{j}}^{K}(z) \leq C e^{\varepsilon \operatorname{deg} p_{j}} \Phi_{\operatorname{deg} p_{j}}^{K}(z) \leq C e^{\varepsilon N} \Phi_{N}^{K}(z), \quad \text { for } j \leq d(N) .
$$


Therefore,

$$
S_{N}(z, z)=\sum_{j=1}^{d(N)}\left|p_{j}(z)\right|^{2} \leq d(N) C^{2} e^{2 \varepsilon N} \Phi_{N}^{K}(z)^{2}
$$

Lemma 3.4. Under the hypotheses of Theorem 3.1, we have

$$
\frac{1}{2 N} \log S_{N}(z, z) \rightarrow V_{K}(z)
$$

uniformly on compact subsets of $\mathbb{C}^{m}$.

Proof. Let $\varepsilon>0$ be arbitrary. Recalling that $d(N)=\left(\begin{array}{c}N+m \\ m\end{array}\right)$, we have by Lemma 3.3,

$$
-\frac{m}{N} \log (N+m) \leq \frac{1}{N} \log \left(\frac{S_{N}(z, z)}{\Phi_{N}^{K}(z)^{2}}\right) \leq \frac{\log C}{N}+\varepsilon+\frac{m}{N} \log (N+m) .
$$

Since $\varepsilon>0$ is arbitrary, we then have

$$
\frac{1}{N} \log \left(\frac{S_{N}(z, z)}{\Phi_{N}^{K}(z)^{2}}\right) \rightarrow 0
$$

The conclusion follows from Lemma 3.2 and (17).

Remark: The asymptotic behavior of the orthonormal polynomials $\left\{p_{j}\right\}$ was first studied by A. Zeriahi [Ze], who showed that

$$
\limsup _{j \rightarrow \infty} \frac{1}{\operatorname{deg} p_{j}} \log \left|p_{j}(z)\right|=V_{K}(z), \quad \text { for all } z \in \mathbb{C}^{m} \backslash \widehat{K},
$$

where $\widehat{K}$ denotes the polynomially convex hull of $K$. Zeriahi's formula (18) follows immediately from Lemma 3.4.

Proof of Theorem 3.1: It follows from Lemma 3.4, together with continuity of the complex Monge-Àmpere operator under uniform limits [BT1], that

$$
\left(\frac{i}{2 \pi N} \partial \bar{\partial} \log S_{N}(z, z)\right)^{k} \rightarrow\left(\frac{i}{\pi} \partial \bar{\partial} V_{K}(z)\right)^{k} \quad \text { weak }{ }^{*}
$$

The conclusion then follows from Proposition 2.1.

Example 3.5. Let $K$ be the unit polydisk in $\mathbb{C}^{m}$. Then $V_{K}=\max _{j=1}^{m} \log ^{+}\left|z_{j}\right|$, the Silov boundary of $K$ is the product of the circles $\left|z_{j}\right|=1(j=1, \ldots, m)$, and $d \mu_{e q}=\left(\frac{1}{2 \pi}\right)^{m} d \theta_{1} \cdots d \theta_{m}$ where $d \theta_{j}$ is the angular measure on the circle $\left|z_{j}\right|=1$.

The monomials $z^{J}:=z_{1}^{j_{1}} \cdots z_{m}^{j m}$, for $|J| \leq N$, form an orthonormal basis for $\mathcal{P}_{N}$. A random polynomial in the ensemble is of the form

$$
f(z)=\sum_{|J| \leq N} c_{J} z^{J}
$$

where the $c_{J}$ are independent complex Gaussian random variables of mean zero and variance one. By Theorem 3.1, $\mathbf{E}_{\gamma_{N}^{m}}\left(Z_{f_{1}, \ldots, f_{m}}\right) \rightarrow\left(\frac{1}{2 \pi}\right)^{m} d \theta_{1} \cdots d \theta_{m}$ weak $^{*}$, as $N \rightarrow \infty$. In particular, the common zeros of $m$ random polynomials tend to the product of the unit circles $\left|z_{j}\right|=1$ for $j=1, \ldots, m$. 
Example 3.6. Let $K$ be the unit ball $\{\|z\| \leq 1\}$ in $\mathbb{C}^{m}$. Then the Silov boundary of $K$ is its topological boundary $\{\|z\|=1\}, V_{K}(z)=\log ^{+}\|z\|$, and $\mu_{e q}$ is the invariant hypersurface measure on $\|z\|=1$ normalized to have total mass one.

\section{Scaling limit zero density for orthogonal polynomials on $S^{2 m-1}$}

Examples 3.5 and 3.6 both reduce to the unit disk in the one variable case. In that case, detailed scaling limits are known (see, for example, [IZ]). For a more general compact set $K \subset \mathbb{C}$ with an analytic boundary, scaling limits are found in [SZ2].

In this section, we consider the case where $K=\left\{z \in \mathbb{C}^{m}:\|z\| \leq 1\right\}$ is the unit ball and $\mu$ is its equilibrium measure, i.e. invariant measure on the unit sphere $S^{2 m-1}$. We have the following scaling asymptotics for the expected distribution of zeros of $m$ random polynomials orthonormalized on the sphere:

Theorem 4.1. Let $\left(\mathcal{P}_{N}^{m}, \gamma_{N}^{m}\right)$ denote the ensemble of $m$-tuples of i.i.d. Gaussian random polynomials of degree $\leq N$ with the Gaussian measure $d \gamma_{N}$ induced by $L^{2}\left(S^{2 m-1}, \mu\right)$, where $\mu$ is the invariant measure on the unit sphere $S^{2 m-1} \subset \mathbb{C}^{m}$. Then

where

$$
\mathbf{E}_{\gamma_{N}^{m}}\left(Z_{f_{1}, \ldots, f_{m}}\right)=D_{N}\left(\log \|z\|^{2}\right)\left(\frac{i}{2} \partial \bar{\partial}\|z\|^{2}\right)^{m}
$$

$$
\begin{aligned}
\frac{1}{N^{m+1}} D_{N}\left(\frac{u}{N}\right)=\frac{1}{\pi^{m}} F_{m}^{\prime \prime}(u) F_{m}^{\prime}(u)^{m-1} & +O\left(\frac{1}{N}\right), \\
F_{m}(u) & =\log \left[\frac{d^{m-1}}{d u^{m-1}}\left(\frac{e^{u}-1}{u}\right)\right] .
\end{aligned}
$$

Proof. We write

$$
z^{J}=z_{1}^{j_{1}} \cdots z_{m}^{j_{m}}, \quad z=\left(z_{1}, \ldots, z_{m}\right), J=\left(j_{1}, \ldots, j_{m}\right) .
$$

An easy computation yields

$$
\int_{S^{2 m-1}}\left|z^{J}\right|^{2} d \mu(z)=\frac{(m-1) ! j_{1} ! \cdots j_{m} !}{(|J|+m-1) !}=\frac{1}{\left(\begin{array}{c}
|J|+m-1 \\
m-1
\end{array}\right)\left(\begin{array}{c}
|J| \\
J
\end{array}\right)}
$$

where

$$
|J|=j_{1}+\cdots+j_{m}, \quad\left(\begin{array}{c}
|J| \\
J
\end{array}\right)=\frac{|J| !}{j_{1} ! \cdots j_{m} !} .
$$

Thus an orthonormal basis for $\mathcal{P}_{N}$ on $S^{2 m-1}$ is:

$$
\varphi_{J}(z)=\left(\begin{array}{c}
|J|+m-1 \\
m-1
\end{array}\right)^{\frac{1}{2}}\left(\begin{array}{c}
|J| \\
J
\end{array}\right)^{\frac{1}{2}} z^{J}, \quad|J| \leq N .
$$

We have

$$
\begin{aligned}
S_{N}(z, z)=\sum_{|J| \leq N}\left|\varphi_{J}(z)\right|^{2} & =\sum_{k=0}^{N}\left(\begin{array}{c}
k+m-1 \\
m-1
\end{array}\right) \sum_{|J|=k}\left(\begin{array}{l}
k \\
J
\end{array}\right)\left|z_{1}\right|^{2 j_{1}} \cdots\left|z_{m}\right|^{2 j_{m}} \\
& =\sum_{k=0}^{N}\left(\begin{array}{c}
k+m-1 \\
m-1
\end{array}\right)\|z\|^{2 k} .
\end{aligned}
$$


Hence

$$
S_{N}(z, z)=g_{N}\left(\|z\|^{2}\right), \quad \text { where } g_{N}(x)=\sum_{k=0}^{N}\left(\begin{array}{c}
k+m-1 \\
m-1
\end{array}\right) x^{k}
$$

We note that

$$
g_{N}=\frac{1}{(m-1) !} G_{N}^{(m-1)}, \quad \text { where } G_{N}(x)=\frac{1-x^{N+m}}{1-x} .
$$

We denote by $O\left(\frac{1}{N}\right)$ any function $\lambda(N, u)=\lambda_{N}(u): \mathbb{Z}^{+} \times \mathbb{R} \rightarrow \mathbb{R}$ satisfying:

$$
\forall R>0, \forall j \in \mathbb{N}, \exists C_{R j} \in \mathbb{R}^{+} \text {such that } \sup _{|u|<R}\left|\lambda_{N}^{(j)}(u)\right|<\frac{C_{R j}}{N} .
$$

We note that

$$
N \log \left(1+\frac{u}{N}\right)=u+u^{2} O\left(\frac{1}{N}\right) \quad(\text { for }|u|<N),
$$

and hence

$$
\left(1+\frac{u}{N}\right)^{N}=e^{u}+u^{2} O\left(\frac{1}{N}\right)
$$

Thus we have

$$
\frac{1}{N} G_{N}\left(1+\frac{u}{N}\right)=\frac{e^{u}-1}{u}+O\left(\frac{1}{N}\right)
$$

Hence

$$
\frac{1}{N^{m}} g_{N}\left(1+\frac{u}{N}\right)=\frac{1}{(m-1) !} \frac{d^{m-1}}{d u^{m-1}}\left(\frac{e^{u}-1}{u}\right)+O\left(\frac{1}{N}\right) .
$$

Therefore

$$
\log \left[\frac{(m-1) !}{N^{m}} g_{N}\left(1+\frac{u}{N}\right)\right]=F_{m}(u)+O\left(\frac{1}{N}\right),
$$

where $F_{m}$ is given in the statement of the theorem.

Since the zero distribution is invariant under the $\mathrm{SO}(2 m)$-action on $\mathbb{C}^{m}$, we can write

$$
\mathbf{E}_{\gamma_{N}^{m}}\left(Z_{f_{1}, \ldots, f_{m}}\right)=D_{N}\left(\log \|z\|^{2}\right)\left(\frac{i}{2} \partial \bar{\partial}\|z\|^{2}\right)^{m}
$$

Then $D_{N}\left(\frac{u}{N}\right)$ is the density at the point

$$
z^{N}:=\left(\frac{1}{\sqrt{m}} e^{u / 2 N}, \ldots, \frac{1}{\sqrt{m}} e^{u / 2 N}\right) \in \mathbb{C}^{m}, \quad\left\|z^{N}\right\|^{2}=e^{u / N} .
$$

We shall compute using the local coordinates $\zeta_{j}=\rho_{j}+i \theta_{j}=\log z_{j}$. Let

$$
\Omega=\left(\frac{i}{2} \partial \bar{\partial} \sum\left|\zeta_{j}\right|^{2}\right)^{m}
$$

By Proposition 2.1 and (21), we have

$$
\mathbf{E}_{\gamma_{N}^{m}}\left(Z_{f_{1}, \ldots, f_{m}}\right)=\left(\frac{1}{2 \pi}\right)^{m} \operatorname{det}\left(\frac{1}{2} \frac{\partial^{2}}{\partial \rho_{j} \partial \rho_{k}} \log g_{N}\left(\sum e^{2 \rho_{j}}\right)\right) \Omega
$$


We note that

$$
\Omega=m^{m}\left[1+O\left(\frac{1}{N}\right)\right]\left(\frac{i}{2} \partial \bar{\partial}\|z\|^{2}\right)^{m} \quad \text { at the point } z^{N} .
$$

We let 1 denote the $m \times m$ matrix all of whose entries are equal to 1 (and we let $I$ denote the $m \times m$ identity matrix). By (25) and (27)-(28), we have

$$
\begin{aligned}
D_{N}\left(\frac{u}{N}\right)= & \left(\frac{m}{2 \pi}\right)^{m}\left[1+O\left(\frac{1}{N}\right)\right] \\
& \times \operatorname{det}\left(2 m^{-2} e^{2 u / N}\left(\log g_{N}\right)^{\prime \prime}\left(e^{u / N}\right) \mathbf{1}+2 m^{-1} e^{u / N}\left(\log g_{N}\right)^{\prime}\left(e^{u / N}\right) I\right) \\
= & \frac{1}{\pi^{m}}\left[1+O\left(\frac{1}{N}\right)\right] \operatorname{det}\left(m^{-1} N^{2} F_{m}^{\prime \prime}(u) \mathbf{1}+N F_{m}^{\prime}(u) I\right) .
\end{aligned}
$$

Therefore,

$$
\begin{aligned}
\frac{1}{N^{m+1}} D_{N}\left(\frac{u}{N}\right)= & \frac{1}{N^{m+1} \pi^{m}}\left[1+O\left(\frac{1}{N}\right)\right] \\
& \times\left\{\left[N F_{m}^{\prime}(u)\right]^{m}+m\left[m^{-1} N^{2} F_{m}^{\prime \prime}(u)\right]\left[N F_{m}^{\prime}(u)\right]^{m-1}\right\} \\
= & \frac{1}{\pi^{m}} F_{m}^{\prime \prime}(u) F_{m}^{\prime}(u)^{m-1}+O\left(\frac{1}{N}\right) .
\end{aligned}
$$

Remark: $\quad$ There is a similarity between the scaling asymptotics of Theorem 4.1 and that of the one-dimensional $\mathrm{SU}(1,1)$ ensembles in $[\mathrm{BR}]$ with the norms $\left\|z^{j}\right\|=$ $\left(\begin{array}{c}L-1+j \\ j\end{array}\right)^{-1 / 2}$, for $L \in \mathbb{Z}^{+}$. Then the expected distribution of zeros of random $\mathrm{SU}(1,1)$ polynomials of degree $N$ has the asymptotics [BR, Th. 2.1]:

$$
\mathbf{E}_{N}\left(Z_{f}\right)=\widetilde{D}_{N}\left(\log |z|^{2}\right) \frac{i}{2} d z \wedge d \bar{z},
$$

where (in our notation)

$$
\frac{1}{N^{2}} \widetilde{D}_{N}\left(\frac{u}{N}\right)=\frac{1}{\pi} F_{L-1}^{\prime \prime}(u)+O\left(\frac{1}{N}\right) .
$$

\section{References}

[BT1] E. Bedford and B. A. Taylor, A new capacity for plurisubharmonic functions, Acta Math. 149 (1982), 1-40.

[BT2] E. Bedford and B. A. Taylor, Fine topology, Silov boundary and $\left(d d^{c}\right)^{n}$, J. Funct. Anal. 72 (1987), 225-251.

[Be] R. Berman, Bergman kernels, random zeroes and equilibrium measures for polarized pseudoconcave domains, preprint 2006, math.CV/0608226.

[BR] P. Bleher and R. Ridzal, SU(1,1) random polynomials, J. Statist. Phys. 106 (2002), 147171.

[BSZ1] P. Bleher, B. Shiffman and S. Zelditch, Poincaré-Lelong approach to universality and scaling of correlations between zeros, Comm. Math. Phys. 208 (2000), 771-785.

[BSZ2] Universality and scaling of correlations between zeros on complex manifolds, Invent. Math. 142 (2000), 351-395.

[Bl1] T. Bloom, Orthogonal polynomials in $\mathbb{C}^{n}$, Indiana Univ. Math. J. 46 (1997), 427-452. 
[B12] , Random polynomials and Green functions, Int. Math. Res. Not. 2005 (2005), 1689-1708.

[B13] _ Random polynomials and (pluri)potential theory, preprint 2006.

[BL] T. Bloom and N. Levenberg, Capacity convergence results and applications to a BernsteinMarkov inequality, Trans. Amer. Math. Soc. 351 (1999), 4753-4767.

[DS] T.-C. Dinh and N. Sibony, Distribution des valeurs de transformations méromorphes et applications, Comment. Math. Helv. 81 (2006), 221-258.

[EK] A. Edelman and E. Kostlan, How many zeros of a random polynomial are real? Bull. Amer. Math. Soc. 32 (1995), 1-37.

[Ha] J. H. Hammersley, The zeros of a random polynomial. Proceedings of the Third Berkeley symposium on Mathematical Statistics and Probability, 1954-55, vol II, University of California Press, California 1956, pp. 89-111.

[IZ] I. Ibragimov and O. Zeitouni, On roots of random polynomials, Trans. Amer. Math. Soc. 349 (1997), 2427-2441.

[Kl] M. Klimek, Pluripotential Theory, London Math. Soc. Monographs, New Series 6, Oxford University Press, New York, 1991.

[Le] N. Levenberg, Monge-Ampère measures associated to extremal plurisubharmonic functions in $\mathbf{C}^{n}$, Trans. Amer. Math. Soc. 289 (1985), 333-343.

[NZ] T. V. Nguyen and A. Zériahi, Famille de polynômes presque partout bornées, Bull. Sci. Math., Paris, 107 (1983), 81-91.

[Ra] T. Ransford, Potential Theory in the Complex Plane, London Mathematical Society Student Texts 28, Cambridge University Press, 1995.

[SV] L. A. Shepp and R. J. Vanderbei, The Complex zeros of random polynomials, Trans. Amer. Math. Soc. 347 (1995), 4365-4384.

[Sh] B. Shiffman, Convergence of random zeros on complex manifolds, preprint 2007.

[SZ1] B. Shiffman and S. Zelditch, Distribution of zeros of random and quantum chaotic sections of positive line bundles, Commun. Math. Phys. 200 (1999), 661-683.

[SZ2] - Equilibrium distribution of zeros of random polynomials, Int. Math. Res. Not. 2003 (2003), 25-49.

[SZ3] Landom polynomials with prescribed Newton polytope, J. Amer. Math. Soc. 17 (2004), 49-108.

[SZ4] _ Number variance of random zeros on complex manifolds, Geom. Funct. Anal., to appear

[Si1] J. Siciak, Extremal plurisubharmonic functions in $\mathbf{C}^{n}$, Ann. Polon. Math. 39 (1981), 175211

[Si2] _ Extremal Plurisubharmonic Functions and Capacities in $\mathbf{C}^{n}$, Sophia Kokyuroku in Mathematics, No. 14, Sophia Univ., Tokyo, 1982.

[Za] V. P. Zaharjuta, Extremal plurisubharmonic functions, orthogonal polynomials, and the Bernstein-Walsh theorem for functions of several complex variables (Russian), Ann. Polon. Math. 33 (1976/77), 137-148.

[Ze] A. Zériahi, Capacité, constante de Čebyšev et polynômes orthogonaux associés à un compact de $\mathbf{C}^{n}$, Bull. Sci. Math. 109 (1985), 325-335.

Department of Mathematics, University of Toronto, Toronto, On, CANADA M5S 3G3

E-mail address: bloom@math.toronto.edu

Department of Mathematics, Johns Hopkins University, Baltimore, MD 21218, USA

E-mail address: shiffman@math.jhu.edu 